\title{
Research on Smart Cast for Fish Cage Aquaculture based on image processing
}

\author{
Liyong $\mathrm{Hu}^{1,2, a}$, Di Zheng ${ }^{1, b}$, Feng Qiao ${ }^{1,2, \mathrm{c}}$, Jianming Zhan ${ }^{1}$, Junhua Chen ${ }^{1}$ \\ ${ }^{1}$ Ningbo Institute of Technology, Zhejiang University, Ningbo 315100, P.R. China \\ 2 Ningbo University, Ningbo 315211, P.R. China \\ aemail: huliyong@nbu.edu.cn, bemail: zhengdi@nbu.edu.cn, cemail: qiaofeng5477@163.com
}

Keywords: Fish cage aquaculture; Image processing; Feature extraction; Smart cast

\begin{abstract}
In recent years, with the development of fish cage aquaculture technology, the traditional way of bait casting cannot meet the growing demands any more. As fishes feed with certain regularities, it is important to acquire the feeding conditions of the fishes. Through analyzing the images of fishes feed by the way of image processing, the feature of the shape and the area of fishes is extracted. The gross of feeding is setup based on the shape, which reflects the weight of fish. The feeding amount is changed with the variation of the image of fish in feeding at real time. The cast program according the extracted feature and the cage aquaculture experiment is designed to verify the feeding effect. Experimental result shows that the smart cast can setup the bait according to the growing requirement of the fishes. The bait utilization will be increased, and water pollution will be reduced, and the fish quality can be improved as well through the smart cast system.
\end{abstract}

\section{Introduction}

With the development of marine aquaculture technology, the cage aquaculture develops rapidly for its irreplaceable advantages [1]. Currently fish cage aquaculture is often used feeding machine to cast at a certain time as a certain amount, and this manner of feeding process cannot be modified according to the aquaculture conditions, which wastes too much bait. As the aquaculture technology develops, the feeding machine is needed to cast according to the aquaculture environment and the characteristics of raising species [2]. The amount of bait needed in different growth stages of fishes and the aquaculture environment is different, so the feeding machine should adjust according to the specific situation in real-time [3, 4].

To solve the above problems and needs, this paper studies a way of smart cast used in fish cage aquaculture. To study the regularities of fish feeding by the way of image processing, he control scheme is designed to realize the smart cast which can adapt the regularities of fish growth. The smart cast system can save bait and reduce costs. and it can improve fish quality and protect the marine environment.

\section{Research on the regularities of fish feeding}

In the fish cage aquaculture, fish feeding has some regularity. We can study the regularities through processing the image acquired by the camera [5]. First of all, the image acquisition device is designed to get high quality images for the following image processing; Then process the images through filtering, enhancement, segmentation to separate the fishes from the background of water; At last, the feature of shape and area of feeding fish is extracted to study the variation of the fish feeding process.

\section{Image acquisition}

Using industrial camera and computer to realize the image acquisition, and the camera lens is arranged vertically to reduce the influence caused by the angular difference. The camera is mounted on the bracket to reduce the image blur phenomenon caused by the shake [6]. The images captured by camera are transmitted directly to the computer to process and preserve. Due to the large storage space and the fast memory speed, the computer meets the demand of continuous image acquisition. 
Capture the images in the fish breeding base of Ningbo, and the images are showed in Fig. 1.

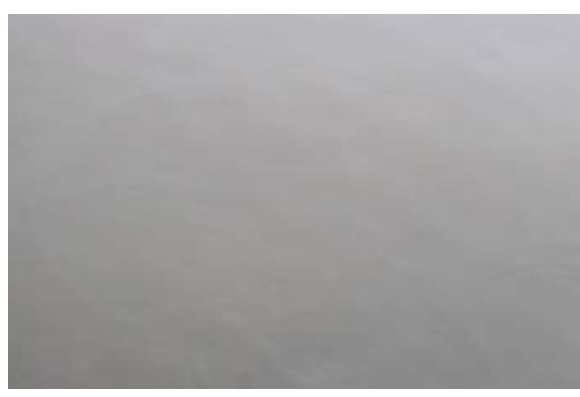

(a) Image before feeding

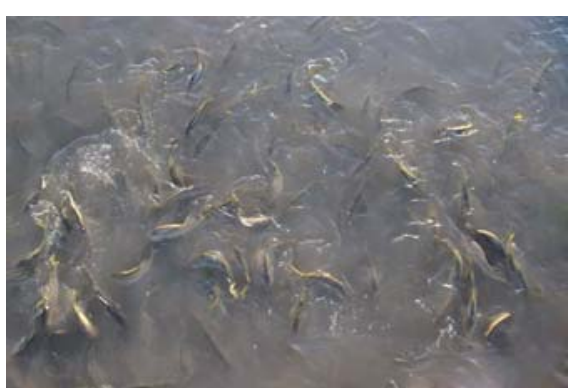

(b) Image in feeding

Fig. 1 The original image

\section{Image processing}

Due to the influence of the environment, the images are mixed with the noise signal, which influents the accuracy of image recognition. First of all, image filtering process should be done to reduce the interference of the noise signal; then study the feature of feeding images through the image enhancement and feature extraction [7].

(1) Filtering: The aim of filtering is to reduce the influence of the noise, we use the way of neighborhood average to filter, and the new value of the pixel is equal to the average of the neighborhood pixel value. The formula is:

$$
\mathrm{g}(x, y)=\frac{1}{M} \sum_{(x, y) \in S} f(x, y)
$$

here, $S$ is the neighborhood of the $\operatorname{pixel}(x, y), M$ is the total pixels of $S$.

(2) Image enhancement: The aim of image enhancement is to enhance the useful feature, and we use the histogram equalization to realize in the time domain. Set the degree of an gray-scale image to $m$, the value of each pixels are $r_{k}(0,1, \cdots, m-1)$, the probability estimation $P_{r}\left(r_{k}\right)$ of the grayscale value is:

$$
\mathrm{P}_{r}\left(r_{k}\right)=\frac{n_{k}}{n}(k=0,1, \mathrm{~m}-1)
$$

here, $n_{k}$ is the total points whose grayscale value is $r k, n$ is the total point of the pixels.

Image enhancement widen the gap which contains more gray-scale pixel and compresses the gap which contains less, thus, the images are enhanced.

(3) Image segmentation: The image segmentation refers the technology of using specific methods to divide the images into a plurality of regions which have specific properties. Usually the image segmentation is based on the discontinuity of gray value or the similarity between pixels. The discontinuity segmentation divides the images based on the discontinuity variation, such as the edge detection, boundary tracking. The similarity segmentation divides the images to some region with similar properties according to the predefined criteria, such as threshold segmentation and regional growth.

To process the feeding images through the above ways and get the binary image through the image segmentation, then detect the fish edge and study the aggregation change regularities of the fishes in the images. The image processing is showed in Fig. 2.

\section{Feature extraction}

In order to find the feeding regularities, we should find out which feature is related to this process. It's very important to select the appropriate feature. If the feature selected is inappropriate, it's hard to guarantee the accuracy of the analysis results. Therefore, it's critical to select target feature in the feature extraction process [8]. 




(a) Image of feeding

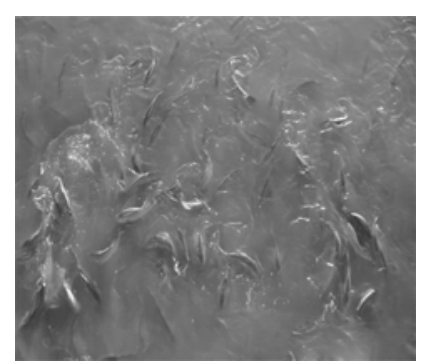

(b) Image filtering

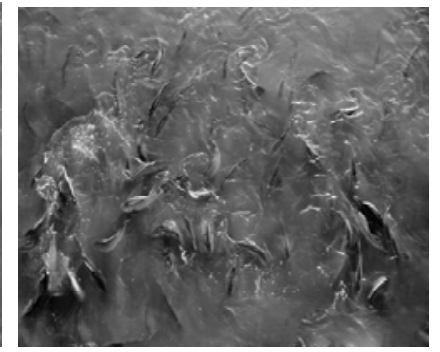

(c) Image enhancement

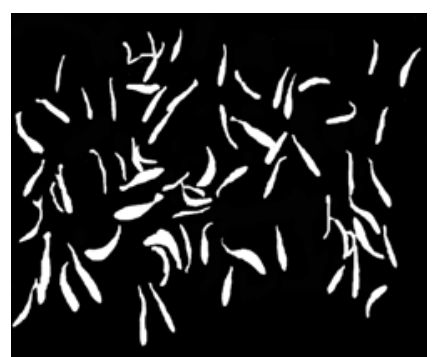

(d) Image segmentation

Fig. 2 Image processing

\section{(1) Extraction of shape feature}

In most object recognition problems, the shape feature can most directly reflect the characteristics of the region of interest. It's a very important parameter in feature extraction process and reflects the geometric characteristics of the target area. After the edge detection, we can directly extract the shape features of the target image region. Chain codes are a common representation in the image recognition mode and can be used to indicate the boundary information of the image. Depending on the slope of the chain, it is divided to 4-direction chain and 8-direction chain codes.

The shape feature of the fish feeding images studied in this paper is complicated, so we use 8-direction chain codes. The numbers of $0,1,2,3,4,5,6$ and 7 are used to represent the eight directions. The even chain codes are horizontal or vertical codes, whose length are 1; and the odd codes are diagonal codes, whose length are $\sqrt{2}$. Therefore, their lengths are fixed and numbers of directions are limited. We often use chain codes to indicate the target boundary.

The shape feature of the feeding fish in the binary image is irregular, and the shape of the fish is a closed irregular curve. Extract the shape feature of the fish and then express in chain codes. The process is showed in Fig. 3.
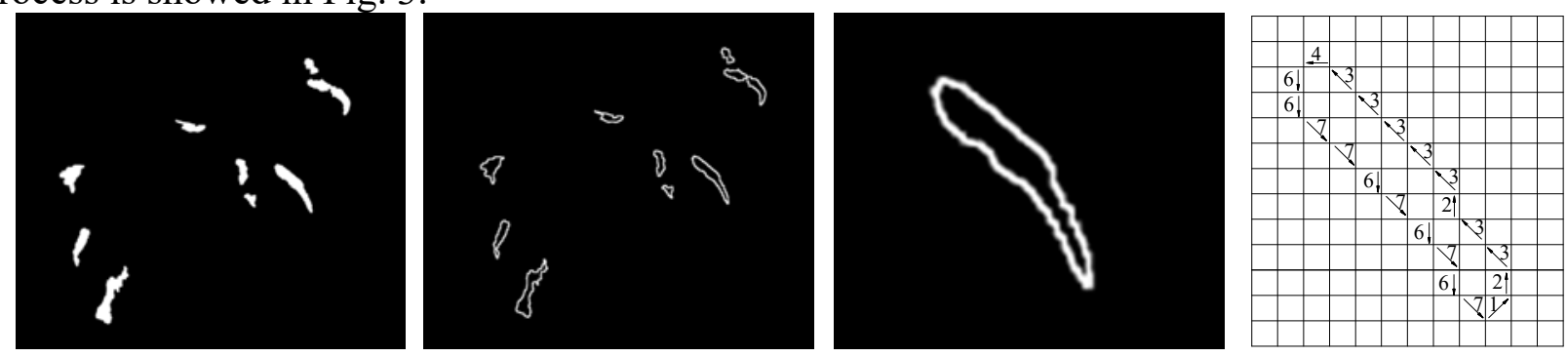

Fig. 3 Chain codes of fish shape

\section{(2) Extraction of area feature}

Fishes are separated from the background in the binary image, the white part represents fishes and black represents water. Using the area feature to analyze the feeding regularities, and the proportion of white pixels in the whole image reflects the degree of aggregation of fish. The increase of the proportion of white pixels means the increase of the fishes which are eating. The variation of the proportion of white pixels in the feeding process is showed in Fig. 4.

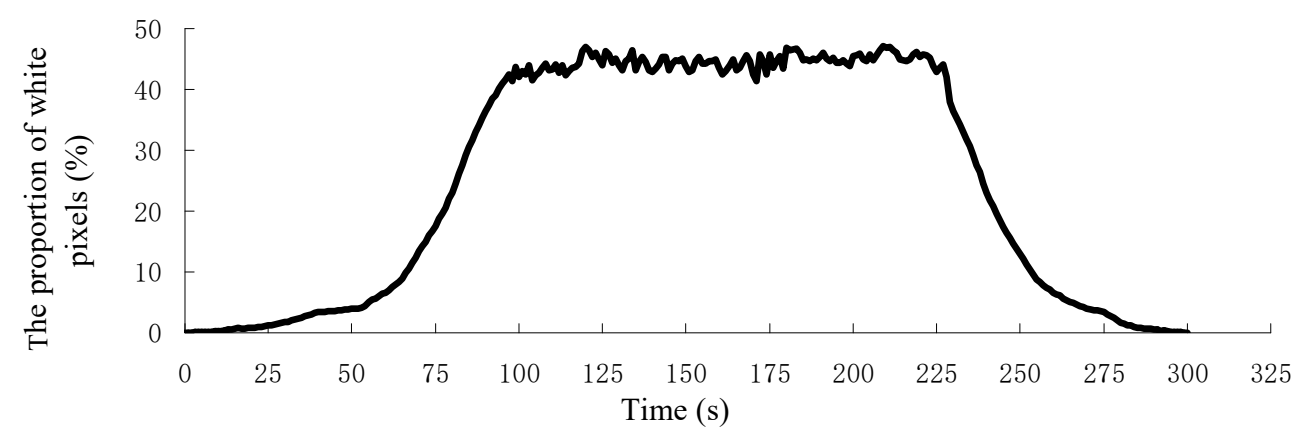

Fig. 4 Variation of the proportion of white pixels in the feeding process

We can know from the Fig. 4 that the proportion of white pixels is changing when the fishes are eating, which indicates that the bait intake is changing. At first, there are a few fishes perceived the 
bait and swim up to eat. As the time goes, more and more fishes swim up to eat, which get dynamic equilibrium within a certain time. Then part of the fishes is no longer hungry and swims down, the aggregation degree decreased. At last, the fishes swim down after getting enough bait, the aggregation degree almost to be zero.

\section{Feeding model}

The feeding process need to consider two factors in the fish cage aquaculture process. Firstly, how much bait is needed in each feeding process, the second is how to cast bait during feeding. According to the image processing results, the shape feature reflects the weight of the fish and can be used to determine the total bait needed. The area feature can be used to decide the bait need at the certain time. Therefore, in the feeding process, calculate the total bait needed through the shape feature firstly then set the ration of casting bait with certain time through the area feature.

\section{1) Determination of the total bait needed}

Usually, the total number of fishes in the cages is fixed and we can calculate the total bait needed through the shape feature. According to the figure 3(d), the position between the fishes and camera lens has a variety of situations, we should choose those which can reflect the characteristics of the shape best to analyze. Extract the chain codes of the fishes in the binary image and calculate the average value, then study the relation between the chain codes and the bait needed [9].

In the aquaculture of large yellow croaker, usually we use the length to study the body features. The relationship between the length of the large yellow croaker and its chain codes is:

$$
L=\frac{\sigma \times l}{2}
$$

In the formula, $L$ is the length of the fish, $\sigma$ is the ratio between the length of fish body and the chain, $l$ is the length of chain codes.

The breeding cycle of the large yellow croaker is so long that the growth characteristics usually are extracted monthly and then modified through the experiment to get the growth characteristics daily. The relationship between the length of the large yellow croaker and the growths days is:

$$
L=-0.026 \times\left(\frac{x}{30}\right)^{2}+1.71 \times\left(\frac{x}{30}\right)+4.15
$$

In the formula, $x$ mean the growths days.

According to the reference [10], the relationship between the weight of the large yellow croaker and the length in the fish cage aquaculture process is:

$$
W=0.0195 \times L^{2.9775}
$$

In the formula, $W$ means the weight of the large yellow croaker.

According to the formula 4 and formula 5, the relationship between the average weight of the large yellow croaker and the growths days is:

$$
W=0.0195 \times\left[-0.026 \times\left(\frac{x}{30}\right)^{2}+1.71\left(\frac{x}{30}\right)+4.15\right]^{2.9775}
$$

If the number of fishes in the cage is $n$, the total amount of bait according to the experiments and aquaculture experience is:

$$
M=0.18 n W
$$

\section{2) Determination of the cast process}

According to the figure 6 , in the feeding process, with the time going, the aggregation of fish exhibits increased firstly and then stabilized finally reduced, so we should cast as this regularity. As we only need to consider the variation of the curve, ignoring the influence of the percent symbol, fit the curve through least squares method and get the function $f(t)$ : 


$$
f(t)= \begin{cases}0.0001 t^{3}-0.0087 t^{2}+0.2355 t-0.7726 & t \in[0,80] \\ -0.0001 t^{3}+0.0011 t^{2}+4.3902 t-259.55 & t \in(80,105) \\ 44.6 & t \in[105,230] \\ -1.1297 t+295.5 & t \in(230,255) \\ 0.0038 t^{2}-2.3117 t+350.3 & t \in[255,300]\end{cases}
$$

Therefore, the feeding process can be decided as follow:

$$
g(t)=\frac{f(t)}{\int_{0}^{300} f(t)} \cdot \lambda M \quad t \in[0,300]
$$

\section{Experiments}

The cast experiment picture is shown in Fig.5. The fish cage is $3 \mathrm{~m} \times 3 \mathrm{~m}$, there are 2000 large yellow croakers in the cage.

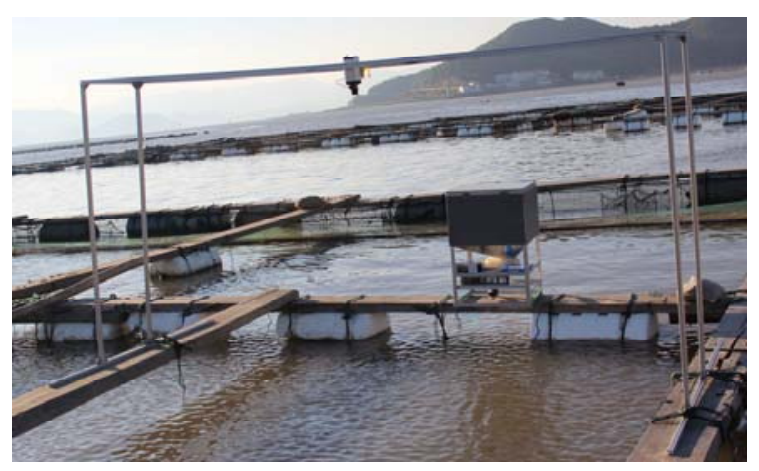

Fig. 5 Cast experiment

Through the shape feature we calculate that the bait needed is $4.5 \mathrm{~kg}$, considering that some bait cannot be eaten and follow into seabed, we design the experiments using $5 \mathrm{~kg}$. We carry out 4 groups of comparative feeding experiments at 5 am and $5 \mathrm{pm}$, the cast time is 5 minute. Firstly, cage 1 and cage 2 use traditional ways, which cast by feeding machine at uniform speed. And then we use smart way on the next day. After the casting, collect the bait which doesn't eat by fishes through the plastic film with holes which lay on the bottom of the cage previously. The experimental result is showed in Table 1.

Table. 1 The experimental contrast between smart way and traditional way of bait casting

\begin{tabular}{|l|c|c|c|c|c|c|}
\hline experiments & $\begin{array}{c}\text { Feeding amount in } \\
\text { traditional way (g) }\end{array}$ & $\begin{array}{c}\text { Remained amount in } \\
\text { traditional way }(\mathrm{g})\end{array}$ & $\begin{array}{c}\text { Feeding amount in } \\
\text { smart way (g) }\end{array}$ & $\begin{array}{c}\text { Remained amount } \\
\text { in smart way }(\mathrm{g})\end{array}$ & $\begin{array}{c}\text { Utilization in } \\
\text { traditional way }\end{array}$ & $\begin{array}{c}\text { Utilization in } \\
\text { smart way }\end{array}$ \\
\hline Cage 1 at $5 \mathrm{am}$ & 5000 & 748 & 5000 & 368 & $85.0 \%$ & $92.6 \%$ \\
\hline Cage 1 at $5 \mathrm{pm}$ & 5000 & 706 & 5000 & 344 & $85.9 \%$ & $93.1 \%$ \\
\hline Cage 2 at $5 \mathrm{am}$ & 5000 & 698 & 5000 & 383 & $86.0 \%$ & $92.3 \%$ \\
\hline Cage 2 at $5 \mathrm{pm}$ & 5000 & 724 & 5000 & 394 & $85.5 \%$ & $92.1 \%$ \\
\hline
\end{tabular}

According to the Table 1 , in the 4 groups of comparative feeding experiments, each cage is casted $5 \mathrm{~kg}$ bait. The average remained bait in traditional way is $719 \mathrm{~g}$ and the average utilization is $85.6 \%$. The average remained bait in smart way is $372.3 \mathrm{~g}$ and the average utilization is $92.56 \%$. The traditional way casts the bait at the same speed and doesn't consider the amount of fishes which are eating, so the bait amount remained is large. The average bait eaten in traditional way is $4821 \mathrm{~g}$, and the average bait eaten in smart way is $4627.7 \mathrm{~g}$, which is $346.7 \mathrm{~g}$ more than traditional way. The experimental results show that the smart cast method improve the utilization of $6.96 \%$, In conclusion, this cast method can save bait and cost. Therefore, the bait utilization is increased; the water pollution is reduced, which meet the need of fish cage aquaculture. 


\section{Conclusion}

(1) According to the existing problems in the fish cage aquaculture and the need to smart cast method, the feeding regularities by the image processing way and the cast method are designed based on the regularities.

(2) In the process of image processing, the shape feature and the area feature through the way of filtering is extracted, enhanced and segmented. The gross of feeding is based on the shape, which reflecting the weight of fish. The feeding amount is changed with the variation of the image of fish in feeding at real time. The shape feature reflects the weight of the fish which can be used to calculate the total bait needed. The variation of the ratio of white pixels reflects the fish feeding regularity and can be use to decide the way to cast. Combining the two features, the smart cast way is designed. The smart system can cast according to the growing requirements of the fishes.

(3) The smart cast method is used in some cage aquaculture experiment. The result shows that this cast method can cast the bait according to the environment and the growing requirements of the fishes. Therefore, the bait utilization is increased; the bait waste and water pollution is reduced, which deserves further improvement and promotion.

\section{Acknowledgement}

In this paper, the research was sponsored by the Major Agriculture Project of Ningbo, Zhejiang Province (Project No. 2015C110015).

\section{References}

[1] B.Chatain, L.Debas, A.Bourdillon. A photographic larval fish counting technique: comparison with other methods, statistical appraisal of the procedure and practical use [J]. Aquaculture, 1995, 141(2): 83-96.

[2] C.M.Chang, W. Fang, R.C.Jao, et al. Development of an intelligent feeding controller for indoor intensive culturing of eel [J]. Aquacultural Engineering, 2005, 32: 343-353.

[3] Nikos Papandroulakis, Papaioannou Dimitris, Divanach Pascal. An automated feeding system for intensive hatcheries [J]. Aquacultural Engineering, 2002, 26: 13-26.

[4] Hui Zhang, Qiwei Wei,Myounghee Kang.Measurement of swimming pattern and body length of cultured Chinese sturgeon by use of imaging sonar [J]. Aquaculture, 2014, 434: 184-187.

[5] Vassilis M.Papadakis, Ioannis E.Papadakis, Fani Lamprianidou. A computer-vision system and methodology for the analysis of fish behavior [J]. Aquacultural Engineering, 2012, 46: 53-59.

[6] Kevin D. Parsonage, Royann J. Petrell. Accuracy of a machine-vision pellet detection system [J]. Aquacultural Engineering, 2003, 29: 109-123.

[7] M.Foster, R.Petrell, M.R.Ito.et al. Detection and Counting of Uneaten Food Pellets in a Sea Cage Using Image Analysis [J]. Aquacultural Engineering, 1995, 14(3): 251-269.

[8] Heng Ma, Tsueng-Fang Tsai, Chia-Cheng Liu. Real-time monitoring of water quality using temporal trajectory of live fish [J]. Expert Systems with Applications, 2010, 37: 5158-5171.

[9] Paul F.Newbury, Philip F.Culverhouse, Derek A. Pilgrim. Automatic fish population counting by artificial neural network [J]. Aquaculture, 1995, 133(1): 45-55.

[10] Chen Hui, Chen Wu, Lin GuoWen, et al. Themorphological characteristics and growth pattern of cage-cultured large yellow croaker(Larimichthys crocea)in Guanjing-Yang population [J]. Marine Fisheries, 2007, 29(4): 331-336.(In Chinese) 